\title{
Obesity - a closer look to cell mechanisms disfunction
}

\author{
Elena BALASESCU ${ }^{1}$, Larisa Diana PANDIA ${ }^{2}$, Roxana loana NEDELCU ${ }^{1}$, \\ Alice BRINZEA ${ }^{1}$, Daniela Adriana ION ${ }^{1}$ \\ 1 "Carol Davila" University of Medicine and Pharmacy, Bucharest, Romania \\ ${ }^{2}$ Astera Diamed Diabetes \& Nutrition Center, Bucharest, Romania
}

\section{ABSTRACT}

Obesity is a complex, multifactorial condition, a major public heal th problem with an increasing prevalence worldwide. Obesity is characterized by an excess of adipose tissue, a low degree of chronic inflammation and disorders in the synthesis of biologically active hormones and peptides which intervene in regulating appetite and energy balance, immunity, insulin sensitivity, angiogenesis, blood pressure, lipid metabolism and homeostasis of the body.

The visceral adipose tissue accumulation is accompanied by metabolic disorders that have as a substrate subclinical inflammation and signaling by intracellular pathways that lead to irreversible cellular structural and functional changes.

The long-term impact of overweight and obesity translates into shortening life expectancy and disability, due to association with severe comorbidities, such as cardiovascular diseases, diabetes, oncological conditions. Therefore, understanding the cellular mechanisms involved in obesity may facilitate the highlighting of new possible therapeutic targets.
\end{abstract}

Keywords: obesity, inflammation, adipocyte, mechanisms, signaling pathways

\section{INTRODUCTION}

It has been established that the prevalence of obesity tripled between 1975 and 2016, with the World Health Organization (WHO) pointing out that approximately 2.8 million people annually die due to overweight and obesity (1). It is estimated that $39 \%$ of the adult population was overweight and $13 \%$ were obese in 2016 (1).

The main complications of obesity and associated pathologies are: cardiovascular (atherosclerosis, ischemic heart disease, myocardial infarction, sudden death, hypertension, stroke, heart failure, deep vein thrombosis, pulmonary embolism etc.), respiratory (respiratory failure, sleep apnea syndrome, pulmonary hypertension etc.), osteoarticular (gonarthrosis etc.), metabolic (insulin resistance, type 2 diabetes, hyperuricemia, gout etc.), digestive (gallstones, hepatic steatosis etc.), neoplasms (in men: prostate, colorectal, in female: breast, ovary, endometrium, cervix), endocrine (hypogynadism, infertility etc.), psychosocial (altered quality of life, altered image and self-esteem, discrimination), obstetric complications, increased operative risk (2).

The result of the interaction between genetic predisposition (thousands of genes have been identified to intervene in genetic predisposition even without playing a decisive role in the occurrence of obesity), environmental factors, socio-economic factors and eating habits, obesity is characterized by an imbalance be- 
tween high and low caloric energy intake, which will lead to the gradual accumulation of adipose tissue. An essential role in the onset of obesity is played by high in calories food and inadequate diet (eating behavior is complex regulated in the central nervous system under both hormonal and psychological influence), s edentary lifestyle (recreational physical inactivity, office work, advanced technology), some drugs (antipsychotics, tricyclic antidepressants, insulin, some oral antidiabetics, corticosteroids). A "chronic relapsing disease process" (3) with an increased risk of mortality and morbidity, obesity is a "continuum" in which life expectancy is reduced by 7 to 10 years in men and women with severe obesity (4).

\section{DEFINITION AND CLASSIFICATION}

The WHO defined and classified the degrees of obesity according to the body mass index (BMI), calculated by the weight/height ${ }^{2}$ ratio. Obesity is defined by a BMI $\geq 30 \mathrm{~kg} / \mathrm{m}^{2}$. Depending on BMI, there are 3 degrees of obesity: $30-34.9 \mathrm{~kg} / \mathrm{m}^{2}$ I degree of obesity, $35-39.9 \mathrm{~kg} /$ $\mathrm{m}^{2}$ II degree of obesity, $\geq 40 \mathrm{~kg} / \mathrm{m}^{2}$ III degree of obesity. The risks related to obesity depend not only on the amount of adipose tissue, but also on its distribution, depending on which we can talk about two types of obesity. First one is abdominal or android obesity, with the disposition of adipose tissue at the subcutaneous or visceral level, the visceral disposition having pathological significance by determining the occurrence of metabolic disorders (insulin resistance, atherogenic dyslipidemia), increased cardiovascular risk regardless of body mass index, and endocrine disorders. The second one is gluteofemoral or gynoid obesity with the arrangement of adipose tissue in the buttocks, hips and thighs. It is more common in women and has a low cardiovascular risk.

\section{MECHANISMS OF OCCURRENCE}

An essential role in the appearance of excess weight is played by genetic predisposition, the intervention of the brain-gut axis (5) and its regulation mechanisms, central intervention of hypothalamus as well as by hormonal (so called peripheral) intervention.

The regulation of the food intake is made in the hypothalamus at the level of the arched nucleus (6). The intervention of the hypothalamus is performed through the orexigenic pathway that expresses hypothalamic neuropeptides involved in leptin actions (such as Agouti-related peptides (AgRP) $(5,7)$, neuropeptide Y (NPY), melanin concentrating hormone and orexins (8)) and the anorexigenic pathway, through proopiomelanocortin (POMC), corticotropin-releasing hormone (CRH).
These pathways are genetically influenced but also can be influenced through signals from the periphery - adipose tissue and digestive tract.

Proopiomelanocortin is a hormone that cleaves into smaller peptides with various roles, which bind with different affinities to homologous receptors (6). At least $3 \%$ of the causes of morbid obesity are due to the existence of a genetic defect (2) that affects the gene of the hypothalamic melanocortin 4 receptor (MC4R), a receptor that plays a major role in regulating eating behavior (9). A syndrome with total proopiomelanocortin deficiency due to POMC gene mutation, characterized by early-onset obesity, adrenal insufficiency, reduced skin pigmentation and red-orange hair, is also cited in the literature (2).

The adipose tissue intervenes through the secretion of adipocytokines (leptin, adiponectin, resistin, adipsin, omentine, TNF $\alpha$, interleukins) in regulating food homeostasis through specific receptors in the hypothalamus. Leptin has a role in diminsh appetite by inhibiting NPY/AgRP neurons and stimulating POMC neurons, but is also involved in inflammation and angiogenesis (8). Congenital leptin deficiency due to a mutation in the leptin gene is characterized by early morbid obesity and is accompanied by hyperphagia and hypogonadotropic hypogonadism. There is a similar form of manifestations, due to a resistance to leptin caused by a defect of the leptin receptor gene, in which its administration is inefficient, the respective patients having even a high serum leptin level (2). It has been demonstrated that leptin influences cell proliferation, angiogenesis (8) and also, in ovarian cancer cells, can inhibits apoptosis through suppression of the cyclin-dependent kinase inhibitor p21 and p27 (10). Physiological, leptin has a central effect acting on POMC neurons where regulates the energy balance through signaling on the PI3K pathway (and thus promoting suppression of food intake and enhancing body energy expenditure) (11). In obesity, systemic inflammation leads to central resistance on leptin action, central inhibition of PI3K and fat gain (11).

The digestive tract secretes a number of appetite-regulating hormones including ghrelin, pancreatic polypeptide, YY peptide, glucagon-like peptide -1 (GLP-1), oxytomodulin and cholecystokinin (7). With the exception of ghrelin (12), which is an orexigenic hormone that causes hunger in the absence of food, all the others are anorexigenic hormones, which cause satiety and decreased appetite. The secretion of these hormones is stimulated or inhibited by food ingestion and physiologically, achieves through complementary effects the regulation of gene expression of neurons in the arcuate nucleus and thus food intake $(5,7)$. 


\section{SPECIFIC TISSUE AND CELL CHANGES}

Obesity is characterized by changes in the mass and functions of adipose tissue due to an increased mechanical, inflammatory and metabolic stress (13). The adipose tissue undergoes a remodeling process that is based on both increasing the number (hyperplasia) and increasing the size of adipocytes (hypertrophy), on the presence of inflammatory infiltrate, high levels of cytokines, tissue damage and healing through fibrosis (13). The mechanisms underlying adipose tissue expansion, adipocyte hypertrophy and their hyperplasia (increased cell turnover through cell proliferation and differentiation, in Akt signaling dependent manner (14)) can also explain their functional changes. Normally, adipocytes are responsive for lipogenesis and storage of lipids and also intervene in control of energy metabolism through adipokine secretion. Abnormal adipocytes express lower insulin-receptors and become resistant to insulin action, increase secretion of promoting inflammation cytokines, accumulates reactive oxygen species - factors that trigger abnormal signaling pathways and lead to a senescent-like pro-inflammatory state (15). Due to a prolonged exposure to different types of stress, different types of cell death pathways can be triggered at the adipocytes level (16). In humans, the normal turnover of adipocyte is approximately $10 \%$ per year (17)) and adipocyte regeneration process can explain accelerating adipogenesis during obesity. Excess accumulation of adipose tissue is accompanied by local hypoxia with impaired functionality and synthesis of substances involved in the local and systemic inflammatory process with the appearance of a vicious circle $(3,12)$; moreover, abnormal adipose tissue become unable to perform its functions.

At cellular level, due to inflammation, increased oxidative stress, production of reactive oxygen species and mitochondrial disfunction, were described early aging processes (15). Beside adipocytes hypertrophy, another major feature of obesity is represented by the macrophages accumulation in adipose tissue, accumulation that is achieved with the help of MCP-1 (chemoattractant protein-1 of the monocyte) (18). The adipocyte-derived factors, hyaluronan and serum amyloid $A$ are also involved in the recruitment of monocytes and the accumulation of macrophages in adipose tissue (19). Serum amyloid A and hyaluronan are components of the matrix, and are involved in increasing the adhesion and retention of monocytes by hypertrophied adipocytes, but also in increasing the chemotaxis of monocytes. Therefore, the hypertrophied adipocyte is accompanied by increased production of serum amyloid $A$ and hyaluronan, which acts synergistically with MCP-1 in the recruitment and adhesion of monocytes with the production of local inflammation (19). Serum amyloid A plays an important role in both local and systemic inflammation (20), through the ability to recruit inflammatory cells and by activating endothelial cells and increasing the expression of adhesion molecules intercellular adhesion molecule-1 (ICAM-1) and vascular cell adhesion molecule 1 (VCAM-1) (21).

Han et al. showed in a study performed both in vitro on cell cultures and in vivo in obese mice (19), an increase in activity (up-regulation) of nuclear factor $\mathrm{kB}$ (NF-kB), an indicator of proinflammatory status, and a decrease in activity (down-regulation) of activated peroxisome proliferation receptors- gamma (PPAR- $\gamma$ ), accompanied by increased expression of serum amyloid $A$ and hyaluronan in hypertrophied adipocytes. Administration of thiazolidinedione, a PPAR- $\gamma$ agonist, determined the reversibility of these changes (19).

The family of PPAR nuclear receptors act as transcription factors, is activated by ligands (such as fatty acids and their derivatives, prostaglandin analogues and leukotrienes, fibrates, NSAIDs, glitazones) and play an essential role in modulating adipogenesis, energy balance, lipid and carbohydrate metabolism. There are 3 subtypes of receptors with tissue distribution and specific metabolic effects: PPAR- $\alpha$ which modulates the expression of genes encoding enzymes and other proteins involved in lipid metabolism and atherogenesis, PPAR- $\beta / \delta$ involved in the reverse cholesterol transport (22) and PPAR- $\psi$.

Metabolic effects of PPAR- $\gamma$ activation are responsible for induction of adipocyte differentiation (preadipocytes express PPAR- - , the receptor level being further increased in the initial stages of adipocyte differentiation), redistribution of adipose tissue (decreases visceral adipose tissue and increases subcutaneous adipose tissue), improving insulin sensitivity by decreasing the cytokine Tumor necrosis factor alpha (TNF $\alpha$ ) and increasing adiponectin expression. Activation of PPAR- $\gamma$ promotes decreased insulin resistance, attenuates hyperinsulinism and improves insulin sensitivity in adipose tissue, liver, skeletal muscle. Improving carbohydrate metabolism by increasing the expression and translocation of glucose transporters (GLUT 1, GLUT 4) to the cell surface, increasing glucose consumption in the liver and skeletal muscle, inhibition of angiogenesis induced by vascular endothelial growth factor (VEGF) and anti-inflammatory effects (by increasing adiponectin secretion and reducing the production of resistance and TNF $\alpha$ ) acts synergistically to an antioxidant effect (reduce the plasma level of chemokines, MCP-1, antifibrinolytic proteins, inhibitor of plasminogen activator 1 , endothelial cell adhesion molecule, C-selectin, ICAM-1. 


\section{ADIPONECTIN,CELLSIGNALINGPATHWAYS AND CELL DEATH}

The pathophysiological mechanisms described above are the basis for the premature onset of programmed cell death. The synthesis of proinflammatory cytokines and the accumulation of reactive oxygen species have the ability to activate the mitogen-activated protein kinase (MAP kinase) signaling pathway (with increased posttranscriptional expression of p27 and other factors such as nuclear factor-KB (NF-KB) and transforming growth factor- $\beta 1$ (TGF- $\beta 1$ ) and the appearance of morphological changes (increased extracellular protein matrix synthesis, cellular hypertrophy). After mitochondrial deterioration and its sensitization to apoptotic stimuli (23), cell enters in the final phase of programmed cell death with activation of proteases that degrade cytoskeletal and nuclear proteins, as well as endonucleases capable of degrading nuclear material with the appearance of lesions typical of apoptotic cells, incompatible with life.

An important role in counteracting the action of inflammatory mediators at the cellular level has adiponectin (ADPN), an adipocytokine synthesized mainly in adipose tissue. Different effects of adiponectin, due to its various forms, explain the stimulation of food intake (ADPN enhances adenosine monophosphate (AMP)-activated protein kinase (AMPK) in the arcuate nucleus of hypothalamus) and promotion of fatty acid oxidation and insulin sensitivity (through the activation of AMPK at peripheral level) (24). There were described high-molecular-weight (HMW-ADPN), middle-molecular-weight (MMW-ADPN), and low-molecular-weight (LMW-ADPN) forms of adiponectin (24), with opposite effects on weight gain: the LMW-ADPN and MMW-ADPN can activate AMPK via adiponectin receptor 1 (AdipoR1) in the hypothalamus, favoring the fat accumulation (increase food intake, reduce energy consumption) (24), while HMW-ADPN activates AMPK in peripheral tissues such as skeletal muscle and stimulates heartburn $(25,26)$.

With multiple roles in cell signaling, involved in increasing sensitivity to insulin action by stimulating the phosphorylation of the insulin receptor, the adiponectin's action is mediated by binding to specific receptors, AdipoR1/adipoR2. AdipoR1 and R2 receptors are predominantly expressed in the liver and muscle and are involved in initiating signal transduction in intracellular signaling, secondary to phosphorylation and activation of AMPK, a central enzyme of homeostasis regulation. Secondary to AMPK activation, fatty acid oxidation increases and their circulatory availability decreases, hepatic glucose production decreases as well as the oxygen reactive species production and mitogen activated protein kinase signaling; moreover, cell proliferation is inhibited. Unlike most adipokines, adiponectin expression and serum concentrations are reduced in obesity and insulin resistance (27).

By cloning adiponectin cellular receptors, they have been confirmed as possible therapeutic targets in conditions related to obesity and metabolic syndrome (26, 27). Decreased high molecular weight adiponectin (HMW ADPN) levels has been shown to be essential and causal in obesity, insulin resistance, and metabolic syndrome; it has been also demonstrate that AdipoR1 and AdipoR2 serve as major receptors in vivo, and AdipoR1 activates the AMP-kinase pathway and AdipoR2 the PPAR- $\alpha$ pathway in the liver (26) with increasing insulin sensitivity and decreasing inflammation. Decreased action of adiponectin and increased MCP-1 underlie the link between insulin resistance and adipokine metabolic syndrome; PPAR- $\gamma$ upregulates HMW ADPN, and PPAR- $\alpha$ upregulates ADPN receptors $(26,27)$.

In obesity, adipose tissue remodeling is closely related to adipocyte death (28). The mechanisms of cell death depend on the cellular context and the stimulus that induces death. The chronic inflammation can trigger a real cascade of events (activation of protein kinase $\mathrm{C}$ (PKC) and NF-KB, stimulation of cell proliferation, increased synthesis of cytokines and reactive oxygen species, impaired insulin signaling, activation of TNFa-mediated signal transduction), reduced cell viability and may initiate cell death.

Adipocyte death is initiated by their hypertrophy process. In obesity, systemic disorders can exacerbate cell death, through activating intimate cell signaling mechanisms (such as protein kinase signaling imbalance, with overuse of the MAPK pathway to the detriment of Phosphoinositide 3-kinase/Akt signaling (PI3K/ Akt). Insulin and leptin receptors signaling involves PI3K/Akt pathway which regulates the metabolism of adipose tissue, glucose metabolism processes, cell proliferation and survival (29).

As a result of imbalance in signaling through MAPK and PI3K/Akt pathways, occurs an overexpression of proteases, activation of the caspase cascade, cytoplasmic structural disorganization with rupture of the cytoskeleton and membrane blebbing, irreversible nuclear events (damage to DNA structures, nuclear damage, condensation) and cell death.

Obesity has as substrate insulin resistance, an abnormal glucose and lipid metabolism. Adipose hypertrophy increases immune cells infiltration and cause insulin resistance with blocking PI3K/Akt - mediated inhibition of lipolysis, increase glucose metabolism, decrease production of free fatty acids, promotes inflammation, cell damage and fibrosis (11). PI3K signaling pathway participate in obesity-associated metabolic disorders, being essential in the insulin-dependent regulation of adipocyte metabolism, recruitment of in- 
flammatory cells and neurohormonal regulation of food intake and energy expenditure (11). An abnormal signaling through PI3K/Akt pathway leads to obesity (PI3K/Akt potentiates growth factor signals) but insulin resistance also exacerbates an abnormal signaling through PI3K/Akt pathway (29) thus creating a vicious circle. Abnormal functions of adipose tissue promote high fat degradation and ectopic accumulation of lipids with increased inflammation and insulin resistance (11).

\section{CONCLUSIONS}

Obesity is characterized by changes in the mass and function of adipose tissue that will have a long-term effect which can lead to disability and greater reduction in life expectancy.

The morphological and functional changes of adipose tissue lead to a senescent-like pro-inflammatory state, energy homeostasis alteration and mitochondrial dysfunction, and can be a trigger for adipocytes premature apoptosis.

Although the mechanisms underlying obesity are not fully understood, there is much evidence that even long-term small changes in lifestyle can improve the metabolic control - which can reduce inflammation, cellular stress, abnormal pathways cellular signaling, the accumulation of profibrotic factors and thus can prevent the installation of irreversible ultrastructural lesions.

Elucidating the pathophysiological pathways in obesity can be a first step in identifying new treatment targets.

\section{Acknowledgement}

All the authors had equal contributions.

Conflict of interest: none declared Financial support: none declared

\section{REFERENCES}

1. http://www.who.int/mediacentre/factsheets/ fs $311 /$ en/.

2. Yosipovitch G, DeVore A, Dawn A. Obesity and the skin: skin physiology and skin manifestations of obesity. J Am Acad Dermatol. 2007;56(6):901-20.

3. Bray GA, Kim KK, Wilding JPH, World Obesity F. Obesity: a chronic relapsing progressive disease process. A position statement of the World Obesity Federation. Obes Rev. 2017;18(7):715-23.

4. Lung T, Jan S, Tan EJ, Killedar A, Hayes A. Impact of overweight, obesity and severe obesity on life expectancy of Australian adults. Int J Obes (Lond). 2019;43(4):782-9.

5. Alhabeeb H, AlFaiz A, Kutbi E, AIShahrani D, Alsuhail A, AIRajhi S, et al. Gut Hormones in Health and Obesity: The Upcoming Role of Short Chain Fatty Acids. Nutrients. 2021;13(2).

6. Biglari N, Gaziano I, Schumacher J, Radermacher J, Paeger L, Klemm P, et al. Functionally distinct POMC-expressing neuron subpopulations in hypothalamus revealed by intersectional targeting. Nat Neurosci. 2021.

7. Adamska E, Ostrowska L, Gorska M, Kretowski A. The role of gastrointestinal hormones in the pathogenesis of obesity and type 2 diabetes. Prz Gastroenterol. 2014;9(2):69-76.

8. Becerril S, Rodriguez A, Catalan V, Ramirez $B$, Unamuno X, Portincasa $P$, et al. Functional Relationship between Leptin and
Nitric Oxide in Metabolism. Nutrients. 2019;11(9).

9. Ayers KL, Glicksberg BS, Garfield AS, Longerich S, White JA, Yang P, et al. Melanocortin 4 Receptor Pathway Dysfunction in Obesity: Patient Stratification Aimed at MC4R Agonist Treatment. J Clin Endocrinol Metab. 2018;103(7):2601-12.

10. Skirnisdottir I, Akerud H, Seidal T, Sundstrom-Poromaa I. Cell Cycle Regulator p27 Mediates Body Mass Index Effects in Ovarian Cancer in FIGO-stages I-II. Cancer Genomics Proteomics. 2019;16(6):443-50.

11. Beretta M, Bauer M, Hirsch E. PI3K signaling in the pathogenesis of obesity: The cause and the cure. Adv Biol Regul. 2015;58:1-15.

12. Cui H, Lopez M, Rahmouni K. The cellular and molecular bases of leptin and ghrelin resistance in obesity. Nat Rev Endocrinol. 2017;13(6):338-51.

13. Wang L, Wang S, Shi Y, Li R, Gunther S, Ong YT, et al. YAP and TAZ protect against white adipocyte cell death during obesity. Nat Commun. 2020;11(1):5455.

14. Rigamonti A, Brennand K, Lau F, Cowan CA. Rapid cellular turnover in adipose tissue. PLoS One. 2011;6(3):e17637.

15. Santos AL, Sinha S. Obesity and aging: Molecular mechanisms and therapeutic approaches. Ageing Res Rev. 2021;67:101268.

16. Eguchi A, Feldstein AE. Adipocyte cell death, fatty liver disease and associated metabolic disorders. Dig Dis. 2014;32(5):579-85.
17. Spalding KL, Arner E, Westermark PO, Bernard S, Buchholz BA, Bergmann O, et al. Dynamics of fat cell turnover in humans. Nature. 2008;453(7196):783-7.

18. Panee J. Monocyte Chemoattractant Protein 1 (MCP-1) in obesity and diabetes. Cytokine. 2012;60(1):1-12.

19. Han CY, Subramanian S, Chan CK, Omer M, Chiba T, Wight TN, et al. Adipocyte-derived serum amyloid $\mathrm{A} 3$ and hyaluronan play a role in monocyte recruitment and adhesion. Diabetes. 2007;56(9):2260-73.

20. Aguilera JJ, Zhang F, Beaudet JM, Linhardt RJ, Colon W. Divergent effect of glycosaminoglycans on the in vitro aggregation of serum amyloid A. Biochimie. 2014;104:70-80.

21. Strissel KJ, Stancheva Z, Miyoshi H, Perfield JW, 2nd, DeFuria J, Jick Z, et al. Adipocyte death, adipose tissue remodeling, and obesity complications. Diabetes. 2007;56(12):2910-8.

22. Marques LR, Diniz TA, Antunes BM, Rossi FE, Caperuto EC, Lira FS, et al. Reverse Cholesterol Transport: Molecular Mechanisms and the Non-medical Approach to Enhance HDL Cholesterol. Front Physiol. 2018;9:526.

23. Hernandez-Aguilera A, Rull A, RodriguezGallego E, Riera-Borrull M, Luciano-Mateo F, Camps J, et al. Mitochondrial dysfunction: a basic mechanism in inflammation-related non-communicable diseases and therapeutic opportunities. Mediators Inflamm. 2013;2013:135698. 
24. Kubota N, Yano W, Kubota T, Yamauchi T, Itoh $\mathrm{S}$, Kumagai $\mathrm{H}$, et al. Adiponectin stimulates AMP-activated protein kinase in the hypothalamus and increases food intake. Cell Metab. 2007;6(1):55-68.

25. Nomura-Nakayama K, Adachi H, Miyatake $\mathrm{N}$, Hayashi N, Fujimoto K, Yamaya H, et al. High molecular weight adiponectin inhibits vascular calcification in renal allograft recipients. PLoS One. 2018;13(5):e0195066.
26. Yamauchi T, Kadowaki T. Physiological and pathophysiological roles of adiponectin and adiponectin receptors in the integrated regulation of metabolic and cardiovascular diseases. Int J Obes (Lond). 2008;32 Suppl 7:S13-8.

27. Kadowaki T, Yamauchi T. Adiponectin and adiponectin receptors. Endocr Rev. 2005;26(3):439-51.
28. Kuroda M, Sakaue H. Adipocyte Death and Chronic Inflammation in Obesity. J Med Invest. 2017;64(3.4):193-6.

29. Huang X, Liu G, Guo J, Su Z. The PI3K/AKT pathway in obesity and type 2 diabetes. Int $J$ Biol Sci. 2018;14(11):1483-96. 